\title{
A Moving Single-Station Doppler Ranging Solution by Means of Direction Finding Method
}

Tao YuP

How to cite this paper: $\mathrm{Yu}, \mathrm{T}$. (2021). A Moving Single-Station Doppler Ranging Solution by Means of Direction Finding Method. World Journal of Electrical and Electronic Engineering, 2(1), 1-10. Retrieved from https://www.scipublications.com/journal/index.php/wjeee/article/view/174

Received: November 01, 2021 Accepted: December 09, 2021 Published: December 10, 2021

Copyright: (c) 2022 by the authors. Submitted for possible open access publication under the terms and conditions of the Creative Commons Attribution (CC BY) license (http://creativecommons.org/licenses /by/4.0/).
China Academy of Management Science, Beijing, China

*Correspondence: $18001648180 @ 163 . c o m$

\begin{abstract}
The Doppler shift is an angle dependent function. Based on the relationship between frequency shift and angle, direction-finding method can be used directly to obtain the ranging solution based on frequency shift measurement. The Doppler ranging solution obtained by this method has excellent calculation accuracy and can keep the same accuracy as the ranging solution based on frequency shift difference processing.
\end{abstract}

Keywords: Single station location; Airborne passive positioning; Doppler shift; Doppler frequency difference; Path difference equation; Direction-finding; Ranging

\section{Introduction}

Existing research results show that the location can be completed based on only one detection by directly using the Doppler change rate[1-3]. Otherwise, if based on Doppler frequency shift measurement, at least two detection are generally required. One of the results of the author's early research on the passive ranging method based on Doppler rate of change is that, based on the mathematical definition, the Doppler rate of change is transformed into the ratio of the Doppler frequency difference to the time difference by using the difference method, and at the same time, by using the velocity vector relationship, an airborne Doppler passive ranging method based on two consecutive frequency shift measurement is derived[4,5]. The result of this study is that there are both advantages and disadvantages. The advantages are that direct measurement of Doppler rate of change is not required. The disadvantages are that two measurements of the Doppler shift are required.

In fact, the Doppler shift is an angle dependent function. From the point of view of pure mathematics, after measuring the frequency shift, the azimuth angle between the movement direction of the detecting platform and the radial distance of the target can be obtained directly according to the frequency shift if the movement speed of the detecting platform and signal wavelength of the detecting target are also known. Based on the relationship between frequency shift and angle, this paper directly uses direction finding method based on trigonometric function to give the ranging solution based on frequency shift measurement. In order to compare the errors more clearly and make it easier for readers to read, the author briefly describes the existing Doppler ranging solutions based on difference processing after the derivation of Doppler ranging solutions by means of direction finding method. The analysis results show that the Doppler ranging solution given by direct direction finding method has excellent calculation accuracy and can keep the same accuracy as the ranging solution based on frequency shift difference processing. 


\section{Doppler ranging solution based on direction finding method}

A Doppler receiver $\mathrm{R}$ is installed on the moving platform to detect the stationary or slow-moving target $\mathrm{T}$ on the ground as shown in Figure 1, and the received Doppler frequency shift is

$$
\lambda f_{d}=v \cos \beta
$$

where: $f_{d}$ is Doppler frequency shift; $\lambda$ the wavelength; $v$ the moving speed of the moving platform; $\beta$ leading angle.

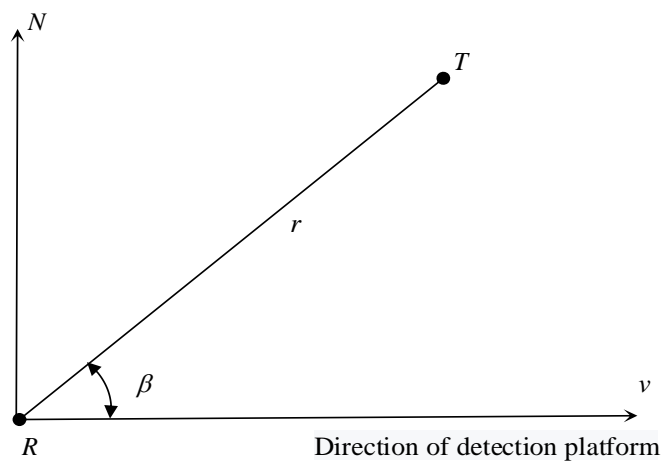

Figure 1. Doppler frequency shift detection of moving single station.

Suppose there is a single station moving in a straight line, as shown in Figure 2, from position 1 to position 2. In this process, Doppler frequency shift detection is performed twice in a row at a single moving station.

$$
\begin{aligned}
& \lambda f_{d 1}=v \cos \beta_{1} \\
& \lambda f_{d 2}=v \cos \beta_{2}
\end{aligned}
$$

In the figure, $d$ represents the moving distance of the sounding station, and $r_{i}$ represents the radial distance between the sounding station and the target.

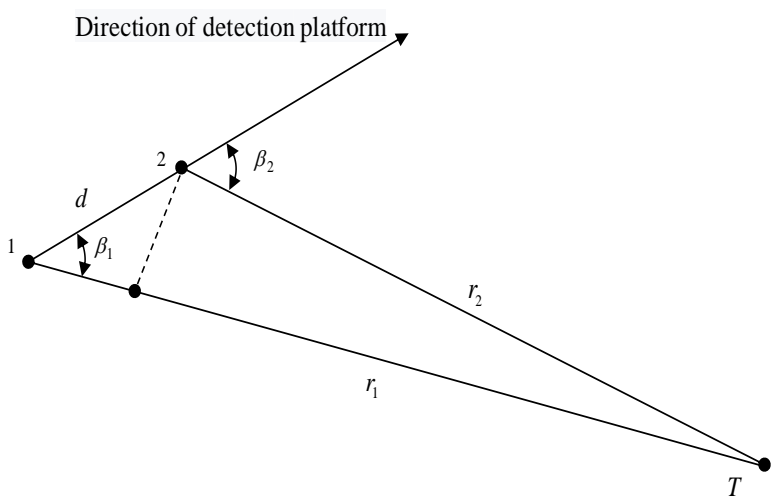

Figure 2. Detection of fixed target by moving single station.

According to the Doppler shift equation (1), the leading angle can be solved directly 


$$
\begin{aligned}
& \beta_{1}=\cos ^{-1}\left(\frac{\lambda}{v} f_{d 1}\right) \\
& \beta_{2}=\cos ^{-1}\left(\frac{\lambda}{v} f_{d 2}\right)
\end{aligned}
$$

According to the geometric relation shown in Figure 2, the sine theorem is used

$$
\frac{d}{\sin \left(\beta_{2}-\beta_{1}\right)}=\frac{r_{1}}{\sin \beta_{2}}=\frac{r_{2}}{\sin \beta_{1}}
$$

The radial distance $r_{2}$ from the detecting platform to the target at the current position

$$
r_{2}=\frac{d \sin \beta_{1}}{\sin \left(\beta_{2}-\beta_{1}\right)}=\frac{d \sin \left[\cos ^{-1}\left(\frac{\lambda}{v} f_{d 1}\right)\right]}{\sin \left[\cos ^{-1}\left(\frac{\lambda}{v} f_{d 2}\right)-\cos ^{-1}\left(\frac{\lambda}{v} f_{d 1}\right)\right]}
$$

\section{Ranging error}

The relative ranging error is analyzed by total differential method. First set

$$
r_{2}=d \frac{P}{Q}
$$

where:

$$
\begin{gathered}
P=\sin \beta_{1} \\
Q=\sin \Delta \beta \\
\Delta \beta=\beta_{2}-\beta_{1} .
\end{gathered}
$$

\subsection{Ranging error resulting from frequency shift measurement}

Ranging error resulting from frequency shift $f_{d 1}$

$$
\frac{\partial r_{2}}{\partial f_{d 1}}=\frac{d}{Q^{2}}\left(Q \frac{\partial P}{\partial f_{d 1}}-P \frac{\partial Q}{\partial f_{d 1}}\right)
$$

Range error resulting from frequency shift $f_{d 2}$

$$
\frac{\partial r_{2}}{\partial f_{d 2}}=\frac{d}{Q^{2}}\left(Q \frac{\partial P}{\partial f_{d 2}}-P \frac{\partial Q}{\partial f_{d 2}}\right)
$$

where:

$$
\frac{\partial P}{\partial f_{d 1}}=\cos \beta_{1} \frac{\partial \beta_{1}}{\partial f_{d 1}}
$$




$$
\begin{gathered}
\frac{\partial P}{\partial f_{d 2}}=0 \\
\frac{\partial Q}{\partial f_{d 1}}=\cos \Delta \beta \frac{\partial \Delta \beta}{\partial f_{d 1}}=\cos \Delta \beta\left(\frac{\partial \beta_{2}}{\partial f_{d 1}}-\frac{\partial \beta_{1}}{\partial f_{d 1}}\right) \\
\frac{\partial Q}{\partial f_{d 2}}=\cos \Delta \beta \frac{\partial \Delta \beta}{\partial f_{d 2}}=\cos \Delta \beta\left(\frac{\partial \beta_{2}}{\partial f_{d 2}}-\frac{\partial \beta_{1}}{\partial f_{d 2}}\right)
\end{gathered}
$$

thereinto

$$
\begin{gathered}
\frac{\partial \beta_{1}}{\partial f_{d 1}}=-\frac{\lambda}{v \sin \beta_{1}} \\
\frac{\partial \beta_{1}}{\partial f_{d 2}}=0 \\
\frac{\partial \beta_{2}}{\partial f_{d 1}}=0 \\
\frac{\partial \beta_{2}}{\partial f_{d 2}}=-\frac{\lambda}{v \sin \beta_{2}}
\end{gathered}
$$

3.2. Ranging error caused by moving distance $d$

$$
\frac{\partial r_{2}}{\partial d}=\frac{\sin \beta_{1}}{\sin \Delta \beta}
$$

\subsection{Ranging error caused by flight speed $v$}

$$
\frac{\partial r_{2}}{\partial v}=\frac{d}{Q^{2}}\left(Q \frac{\partial P}{\partial v}-P \frac{\partial Q}{\partial v}\right)
$$

where:

$$
\begin{gathered}
\frac{\partial P}{\partial v}=\cos \beta_{1} \frac{\partial \beta_{1}}{\partial v} \\
\frac{\partial Q}{\partial v}=\cos \Delta \beta \frac{\partial \Delta \beta}{\partial v}=\cos \Delta \beta\left(\frac{\partial \beta_{2}}{\partial v}-\frac{\partial \beta_{1}}{\partial v}\right)
\end{gathered}
$$

thereinto

$$
\begin{gathered}
\frac{\partial \beta_{1}}{\partial v}=\frac{\lambda f_{d 1}}{v^{2} \sqrt{1-\left(\frac{\lambda}{v} f_{d 1}\right)^{2}}}=\frac{\lambda f_{d 1}}{v^{2} \sin \beta_{1}} \\
\frac{\partial \beta_{2}}{\partial v}=\frac{\lambda f_{d 2}}{v^{2} \sqrt{1-\left(\frac{\lambda}{v} f_{d 2}\right)^{2}}}=\frac{\lambda f_{d 2}}{v^{2} \sin \beta_{2}}
\end{gathered}
$$




\subsection{Computational formula}

When the error of each observation is zero mean, independent of each other, the relative ranging error can be calculated as follows

$$
\sigma_{r}=\frac{1}{r_{2}}\left[\left|\frac{\partial r_{2}}{\partial f_{d 1}}\right| \sigma_{f}+\left|\frac{\partial r_{2}}{\partial f_{d 2}}\right| \sigma_{f}+\left|\frac{\partial r_{2}}{\partial d}\right| \sigma_{d}+\left|\frac{\partial r_{2}}{\partial v}\right| \sigma_{v}\right]
$$

where: $\sigma_{\theta}, \sigma_{f}, \sigma_{d}, \sigma_{v}$ are the root mean square errors of angle, frequency shift, moving distance and flight velocity measurement errors respectively.

The preliminary calculation shows that the root mean square error of moving distance and flight speed measurement error has little influence on the relative ranging error. Therefore, in the following calculation process, only the relative ranging error generated by the frequency shift measurement error is considered

$$
\sigma_{r_{-} D F}=\frac{1}{r_{2}}\left[\left|\frac{\partial r_{2}}{\partial f_{d 1}}\right| \sigma_{f}+\left|\frac{\partial r_{2}}{\partial f_{d 2}}\right| \sigma_{f}\right]
$$

\section{Comparison with the ranging solution based on frequency shift differential pro- cessing}

The simulation results show that the Doppler ranging solution based on the direction finding method has excellent calculation accuracy. At this point, it seems meaningless to analyze the calculation accuracy of different moving distances, different radial distances, different moving speeds and different wavelengths separately. At the same time, the error calculation also shows that the error characteristic of Doppler ranging solution based on direction finding method is basically the same as that of the existing ranging solution based on frequency shift difference processing. Therefore, the relative calculation accuracy and ranging error analysis of Doppler ranging solutions based on direction finding method are put in the section of comparison with the existing ranging solutions based on frequency shift differential processing.

\subsection{Ranging solution of difference based on frequency shift}

This section first retells the mathematical derivation results of previous studies[4,5]. Still based on the geometric model shown in Figure 2, it is assumed that the Doppler change rate detected by the detection platform at position 1 is

$$
\dot{f}_{d}=\frac{v_{t 1}^{2}}{\lambda \cdot r_{1}}
$$

where, $v_{t 1}$ is the tangential velocity of the detection platform; $r_{1}$ the radial distance between the target and the detection platform.

From the mathematical definition (15), the rate of Doppler change can be approximated by the measured value of Doppler frequency difference between two detection endpoints during a time period $\Delta t$ obtained by time difference measuring

$$
\dot{f}_{d}=\frac{\Delta f_{d}}{\Delta t}=\frac{f_{d 2}-f_{d 1}}{\Delta t}
$$

Time difference $\Delta t$ can also be expressed as the ratio of distance traveled to speed traveled 


$$
\Delta t=\frac{d}{V}
$$

By integrating equations (15) and (16), and using the relationship $v^{2}=v_{r}^{2}+v_{t}^{2}$ between velocity vector and its components, and the relationship $v_{r}=\lambda f_{d}$ between radial velocity and Doppler frequency shift, the ranging formula as follows can be obtained

$$
r_{1}=\frac{\left(v^{2}-\lambda^{2} f_{d 1}^{2}\right) \Delta t}{\lambda\left|\Delta f_{d}\right|}=\frac{d\left(v^{2}-\lambda^{2} f_{d 1}^{2}\right)}{v \lambda\left|\Delta f_{d}\right|}
$$

where: $\Delta f_{d}=f_{d 1}-f_{d 2}$.

\subsection{Simulation modification}

Parameter value used in calculation: $r_{1}=600 \mathrm{~km}, d=100 \mathrm{~km}, v=300 \mathrm{~m} / \mathrm{s}$, $\lambda=0.3 m, \sigma_{f}=50 \mathrm{~Hz}$.

The radial distance and Doppler frequency shift values at the two detection positions are respectively selected to calculate. Figure 3 shows some of the calculated curves selectively.

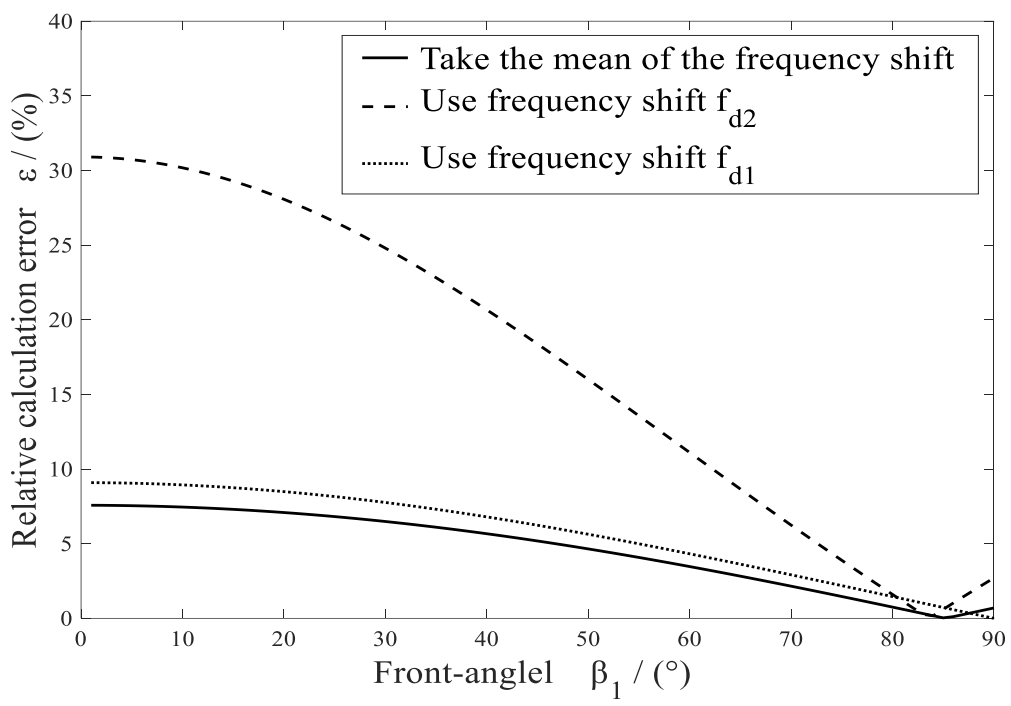

Figure 3. Relative calculation errors of ranging solution using frequency difference directly.

The results show that, assuming that the detection platform moves the distance $d$ uniformly along a straight line, if the average frequency shift at two positions is adopted, the ranging formula at position 1 has the minimum relative calculation error, that is,

$$
r_{1}=\frac{d\left(v^{2}-0.25 \lambda^{2}\left(f_{d 1}+f_{d 2}\right)^{2}\right)}{v \lambda\left|\Delta f_{d}\right|}
$$

The dotted line in Figure 3 shows the relative calculation error of Equation (19). This shows mathematically that there is a lag in the detection of target distance. It is necessary 
to obtain the average value of Doppler frequency shift on a moving distance, and then only the radial distance at the starting position can be accurately obtained.

The dot line in Figure 3 shows the relative calculation error of equation (20), which indicates that the Doppler frequency shift at the terminating position is used to calculate the radial distance $r_{2}$ at position 2, and the relative calculation error obtained is very poor

$$
r_{2}=\frac{d\left(v^{2}-\lambda^{2} f_{d 2}^{2}\right)}{v \lambda\left|\Delta f_{d}\right|}
$$

If equation (20) is simply modified to

$$
r_{2}=\frac{d\left(v^{2}-\lambda^{2} f_{d 1}^{2}\right)}{v \lambda\left|\Delta f_{d}\right|}
$$

The solid line in Figure 3 shows the relative calculation error of Equation (21). The modified results show that, in the case of only two detection, in order to directly use the Doppler shift measurement value at the current moving distance to solve the radial distance $r_{2}$ at the end position more accurately, the Doppler shift value at the starting position 1 should be used to replace the frequency shift value at the second position 2 .

\subsection{Error calculation of frequency shift differential ranging solution}

According to the results of simulation correction, formula (21) is adopted for error analysis. Preset preestablish

$$
r_{2}=a_{0} \frac{P_{0}}{Q_{0}}
$$

where:

$$
\begin{gathered}
a_{0}=\frac{d}{v \lambda} \\
P_{0}=v^{2}-\lambda^{2} f_{d 1}^{2} \\
Q_{0}=\left|\Delta f_{d}\right|
\end{gathered}
$$

Ranging error resulting from frequency shift $f_{d 1}$

$$
\frac{\partial r_{2}}{\partial f_{d 1}}=\frac{a_{0}}{Q_{0}^{2}}\left(Q_{0} \frac{\partial P_{0}}{\partial f_{d 1}}-P_{0} \frac{\partial Q_{0}}{\partial f_{d 1}}\right)
$$

thereinto

$$
\begin{gathered}
\frac{\partial P_{0}}{\partial f_{d 1}}=-2 \lambda^{2} f_{d 1} \\
\frac{\partial Q_{0}}{\partial f_{d 1}}=1 .
\end{gathered}
$$

Ranging error resulting from frequency shift $f_{d 2}$ 


$$
\frac{\partial r_{2}}{\partial f_{d 2}}=-\frac{a_{0} P_{0}}{Q_{0}^{2}} \frac{\partial Q_{0}}{\partial f_{d 2}}
$$

thereinto

$$
\frac{\partial Q_{0}}{\partial f_{d 2}}=1
$$

The relative ranging error caused by frequency shift measurement error

$$
\sigma_{r_{-} F D}=\frac{1}{r_{2}}\left[\left|\frac{\partial r_{2}}{\partial f_{d 1}}\right| \sigma_{f}+\left|\frac{\partial r_{2}}{\partial f_{d 2}}\right| \sigma_{f}\right] \text {. }
$$

\subsection{Comparison of relative calculation accuracy}

During the simulation calculation, the leading angle $\beta_{1}$ at starting point 1 of the detection platform, the radial distance $r_{1}=600 \mathrm{~km}$ from starting point 1 to the target, the moving distance $d=100 \mathrm{~km}$ of the detection platform, the flight speed $v=300 \mathrm{~m} / \mathrm{s}$ and the wavelength $\lambda=0.3 m$ of the detection signal are preset.

Then the radial distance $r_{2}$ of the target at the second position 2 and other geometric parameters are calculated by using trigonometric function relations. According to the definition (1) of Doppler frequency shift, the Doppler frequency shift value is calculated.

Figure 4 shows the relative calculation error curves of Doppler ranging solutions based on direction finding method and existing ranging solutions based on frequency shift measurement. Obviously, the relative calculation error of Doppler ranging solution with direction finding method is almost zero, and the most direct reason is that there is no approximate treatment in the process of proving the ranging solution. In terms of mathematical expression, the leading angle tends to be the same at both probe positions only when the leading angle is small. Now, if you just look at the denominator and it tends to go to zero, it seems to result in a singularity. But in fact, so the leading angle is going to zero, so the numerator is going to zero. Therefore, according to the limit rule of derivative, the whole ranging formula tends to a fixed value.

As far as the shape of the error curve is concerned, the author thinks that the relative error distribution of the Doppler ranging solution by means of direction finding method should be only some chaotic burrs, and has nothing to do with physical characteristics. In other words, there is no functional correlation between the distribution characteristics of the relative calculation error curve and the leading angle in physical characteristics. However, the relative calculation error of the ranging solution based on approximate difference processing is related to the physical characteristics.

According to the mathematical definition (15) of Doppler rate of change, the singularity of the equation does not appear when the tangential velocity and distance are fixed. However, after the approximate difference treatment, the Doppler change rate is directly determined by the difference between the two frequency shifts. According to the definition (1) of Doppler, in the case of fixed velocity, the magnitude of the Doppler shift is only related to the leading angle. Therefore, when the leading angle is small, that is, when the leading angle at the two detection positions tends to be the same, the magnitude of the two frequency shifts is very close, and the difference between the two frequency shifts tends to zero. At this point, the Doppler rate of change tends to be singular, which leads 
to the relative calculation error of the approximate ranging solution is relatively large when the leading angle is small.

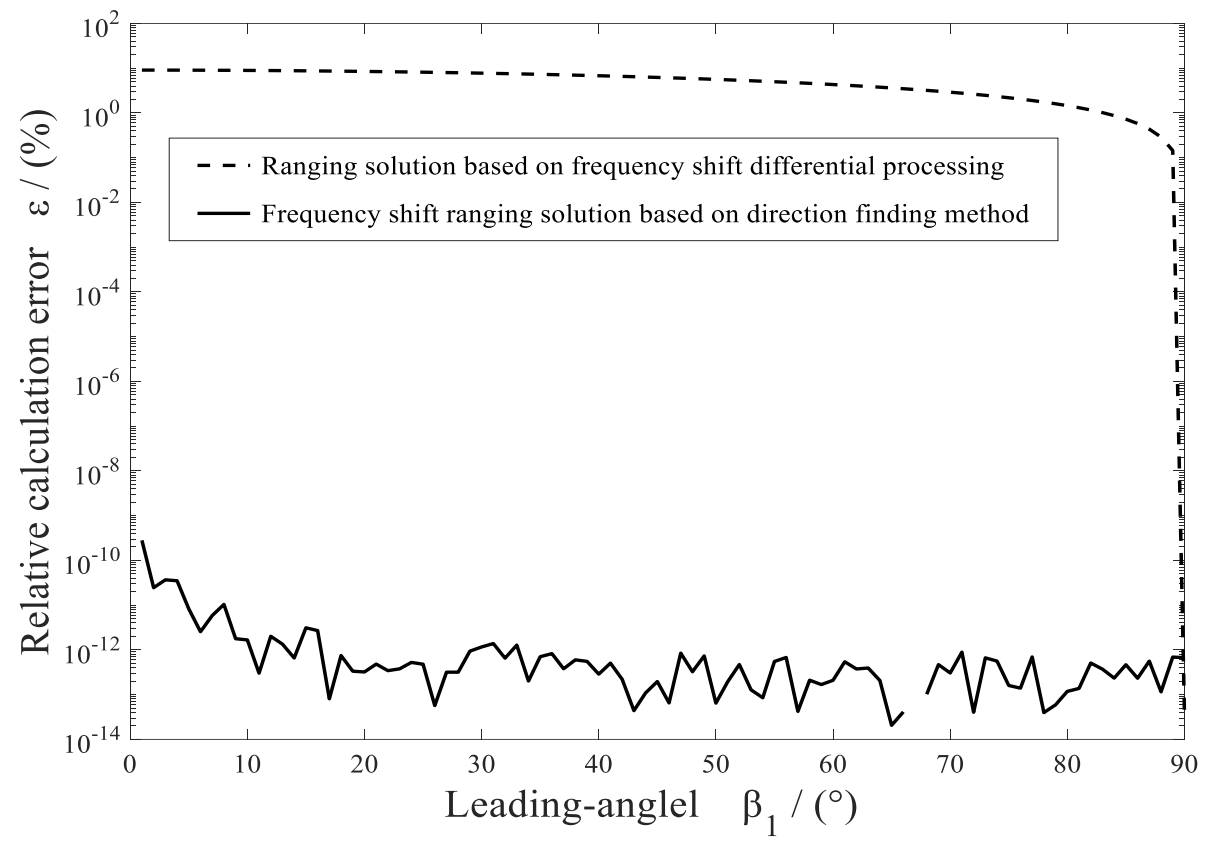

Figure 4. Comparison of computational accuracy.

\subsection{Comparison of ranging errors}

The relative ranging error curves for the two ranging solutions have been shown in Figure 5. When the leading angle is less than 40 degrees, there is a slight difference between the two ranging methods. But when it is greater than 40 degrees and tends to 90 degrees, the relative ranging errors of the two solutions tend to be the same. The author's existing and ongoing research results show that[6-8], under the same frequency shift measurement method, no matter what mathematical deduction method is adopted, the ranging error is basically the same. In other words, the ranging error seems to be only related to the frequency shift measurement method, and has nothing to do with the mathematical expression of the ranging equation. 


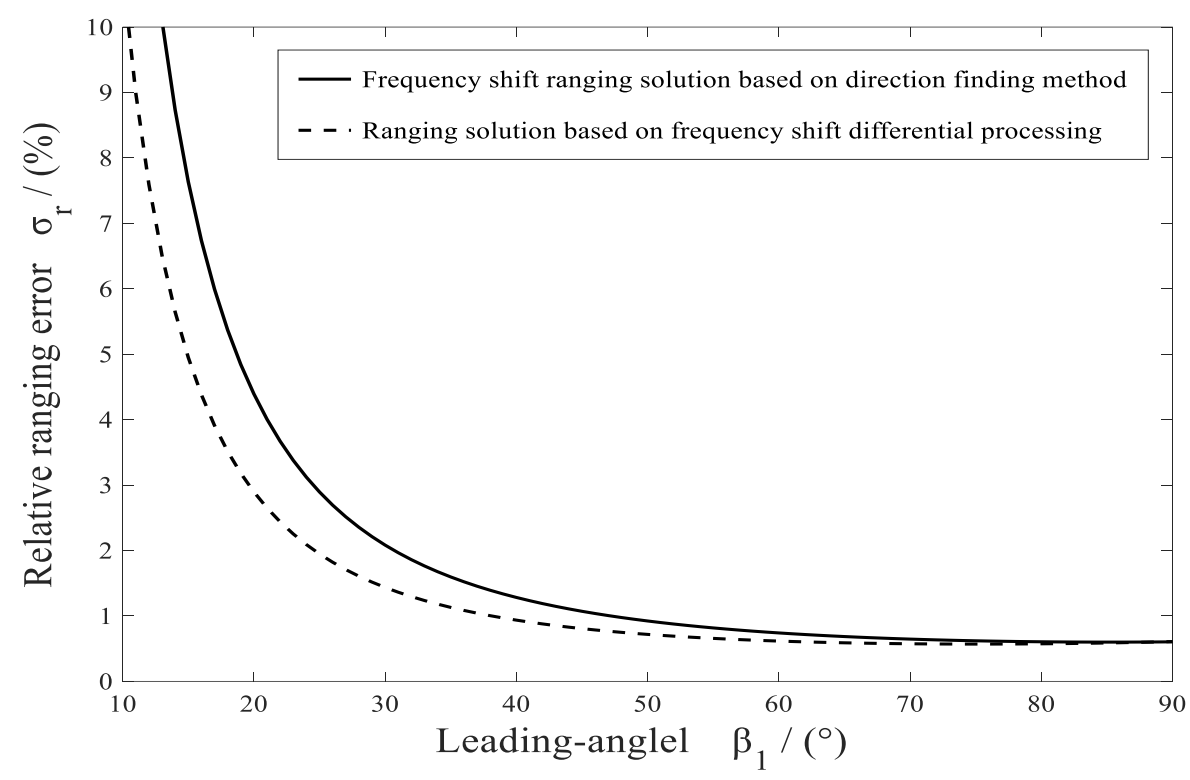

Figure 5. Comparison of relative ranging errors.

\section{Conclusion}

The existing ranging method based on frequency shift differential processing has some approximation in the process of mathematical deduction and verification, so the calculation accuracy of the mathematical formula obtained is relatively poor. Based on the relationship between frequency shift and angle, the Doppler ranging formula derived by direction finding method basically has no approximation. Therefore, the calculation accuracy of the ranging formula obtained is very good. Furthermore, the relative ranging error of the obtained solutions is basically consistent with that of the existing solutions directly based on frequency shift measurement.

Improving the accuracy of formula calculation is not only helpful for engineering design, but also for theoretical research. The research in this paper may provide a reference method for passive ranging technology of motion detection platform.

\section{References}

[1] Li Zong-hua, XiaoYu-qin, Zhou yi-yu, Sun Zhong-kang, "Single-observer passive location and tracking algorithms using frequency and spatial measurements", SYSTEMS ENGINEERING AND ELECTRONICS, vol. 26, no. 5, pp. 613-616, May 2004.

[2] DIAO Ming, WANG Yue, "Research of passive location based on the Doppler changing rate", SYSTEMS ENGINEERING AND ELECTRONICS, vol. 28, no. 5, pp. 696-698, June 2006.

[3] TAN Xin-rong, GAO Xian-jun, LI Bao-zhu, WANG Yu, "An improved tracking filter algorithm of single observer passive localization based on Doppler changing rate", Electronic Design Engineering, vol. 22, no. 8, pp. 77-80, April 2014.

[4] YU Tao, "Airborne ranging principle based on Doppler frequency", INFORMATION AND ELECTRONIC ENGINEERING, vol. 9, no. 1, pp. 22-25, Feb. 2011.

[5] Tao Yu, Technology of Passive detection location, Beijing: National defense industry press, 2017

[6] Tao YU. "An Airborne Passive Positioning Method Based on Angle and Frequency Difference Measurement", 2020 4th International Conference on Electronic Information Technology and Computer Engineering Proceedings, 2020, pp.296-301.

[7] Tao YU. “A Moving Single Station Passive Ranging Method by Interchangeable Relationship between Frequency Shift and Path Difference", To be published.

[8] Tao Yu, "Recursive Equation of Frequency Shift and Its Application in Passive Ranging", To be published. 